\title{
Construcción de un diplomado en estadística aplicada a la investigación: una experiencia en Google Classroom
}

\section{Construction of a diplomat in statistics applied to research: an experience with Google Classroom}

\author{
Joan Chipia \\ joanfernando130885@gmail.com \\ https://orcid.org/0000-0001-6365-8692 \\ Yorman Paredes \\ paredesy@gmail.com \\ https://orcid.org/0000-0002-0319-7641 \\ Universidad de Los Andes, Mérida, Venezuela
}

Recibido: $13 / 06 / 20$

Aceptado: 19/01/21

\begin{abstract}
Resumen
El objetivo fue describir una experiencia educativa sobre la construcción de un Diplomado en Estadística Aplicada a la Investigación con Google Classroom, Facultad de Medicina, Universidad de Los Andes, Mérida, Venezuela, 2018-2020. Método: enfoque cuantitativo, tipo de investigación descriptiva, diseño no experimental, de campo, prospectivo. La muestra fue de 168 participantes en siete cohortes del diplomado. Resultados: $83,3 \%(n=140)$ culminaron el diplomado, el promedio y desviación estándar de calificaciones fue de 16,98 \pm 1,473 puntos. Conclusiones: la construcción del diplomado con la utilización de Google Classroom fue efectiva. El proceso educativo bajo la modalidad b-learning mostró fluidez y permitió sortear dificultades logísticas.
\end{abstract}

Palabras clave: Alfabetización Estadística, B-learning, Educación.

\begin{abstract}
The objective was to describe an educational experience on the construction of a Diplomat in Statistics Applied to Research with Google Classroom, Faculty of Medicine, University of Los Andes, Mérida, Venezuela, 2018-2020. Method: quantitative approach, descriptive research type, non-experimental, field design, prospective. The sample consisted of 168 participants in seven cohorts of the diplomat course. Results: $83.3 \%(n=140)$ completed the diplomat, the average and weighted standard deviation of grades was $16.98 \pm 1.473$ points. Conclusions: the construction of the diplomat with
\end{abstract}

Construcción de un diplomado en estadística aplicada a la investigación: una experiencia en Google Classroom. -
Eduweb, 2021, enero-abril, v.15, n.1./10-18 
the use of Google Classroom was effective. The educational process under the b-learning modality was fluid and allowed to overcome logistical difficulties.

Key words: Statistical Literacy, B-learning, Education.

\section{Introducción}

El artículo de investigación tiene por objetivo describir una experiencia educativa sobre la construcción de un Diplomado en Estadística Aplicada a la Investigación con la utilización de Google Classroom, Facultad de Medicina, Universidad de Los Andes, Mérida, Venezuela, 2018-2020. El Diplomado tiene una duración de 128 horas, divididas en 64 horas presenciales y 64 a distancia. Se estructuró en cuatro (4) módulos: Módulo 1. Conceptos Básicos de Estadística y de Investigación (16 horas); Módulo 2. Estadística Descriptiva (48 horas); Módulo 3. Contraste de hipótesis (48 horas); Módulo 4. Muestreos probabilísticos (16 horas).

El proceso educativo se llevó a cabo en clases teórico-prácticas de manera presencial bajo una teoría de aprendizaje constructivista. Se prepararon actividades didácticas y materiales educativos que se elaboraron en el marco de un modelo instruccional de enseñanza directa, empleando como estrategias docentes la técnica de la pregunta y la resolución de problemas. Se utilizan como herramientas de trabajo los programas Microsoft Excel, Epidat, SPSS para Windows, R y R-Studio. Las actividades a distancia se realizaron con tareas individuales que debían ser entregadas a través de Google Classroom.

La investigación se efectuó con un enfoque cuantitativo, tipo descriptivo, diseño no experimental de campo prospectiva. Se muestran los resultados de las calificaciones de los estudiantes de siete cohortes del Diplomado en Estadística Aplicada a la Investigación. La construcción del Diplomado muestra un promedio de calificaciones y porcentaje de culminación alto. El artículo se divide en cinco (5) partes fundamentales las cuales son: 1) Tema de interés en el que se desarrolla el tema, se explica cómo se planificó el Diplomado, antecedentes y objetivo de la investigación; 2) Método; 3) Resultados; 4) Discusión de resultados; 4) Conclusiones; 5) Recomendaciones.

\section{Tema de interés}

La Estadística es una disciplina que permite suministrar objetividad a los resultados de una investigación, porque a través de indicadores se detalla el tema que se desea explorar, describir, analizar, explicar o proyectar, basado en la recolección y procesamiento de datos, para interpretar y tomar decisiones ante situaciones de incertidumbre en un contexto y tiempo bien definido, buscando ir más allá de la hiperespecialización, fragmentación y compartimentación, las cuales impiden percibir los problemas fundamentales, globales y rompen el tejido complejo de lo real. Para 
Joan Chipia, Yorman Paredes.

construir lo antes señalado es necesaria una reforma del pensamiento de los diferentes actores de proceso investigativo y educativo (Chipia, 2017).

El aprendizaje de la Estadística no solamente está estructurado por una serie de definiciones, procedimientos y técnicas, sino que trata de fomentar un razonamiento crítico, basado en la valoración de evidencia objetiva (Chipia, Cadenas y Lara, 2012). Dado lo señalado anteriormente, para gestionar el aprendizaje de la Estadística se requiere de un proceso planificado, mediante la solución de situaciones problemas, donde el estudiante pueda construir activamente su aprendizaje y adquiera un sentido personal y trascendental en su cotidianidad (Chipia, 2013). Además, el proceso educativo requiere exigencias previas para su desarrollo tales como: disciplina, motivación, habilidades y conocimientos previos, es decir, consultar la documentación adecuada, estas exigencias permitirán el éxito y el aprendizaje de la Estadística (Chipia y Paredes, 2017).

Es oportuno señalar, que para integrar las Tecnologías de la Información y Comunicación (TIC) en una actividad de aprendizaje, es necesario considerar a los participantes y las condiciones específicas donde se van a aplicar, utilizando varios medios de comunicación, porque se genera una mayor relación entre docenteestudiante y entre estudiantes, para buscar el cambio de actitud de un educando pasivo a un discente activo y responsable de su aprendizaje. El docente que plantee actividades de aprendizaje con la utilización de la Web debe transformarse en un facilitador que guía el proceso de enseñanza y aprendizaje, para ello requiere de un mayor compromiso con la labor que está efectuando (Chipia, 2014).

Dadas las consideraciones anteriores se planteó un Diplomado en Estadística Aplicada a la Investigación, considerando el Reglamento de Programas de Formación, Actualización y Capacitación de la Universidad de Los Andes (2011). El diplomado está dirigido a estudiantes de pre y postgrado, profesionales de las diferentes áreas del saber, asesores y tutores de tesis, y todos aquellos interesados en la aplicación de procedimientos estadísticos para el análisis de problemas de interés científico. Se estructuró bajo la modalidad semipresencial (b-learning) con una duración total de 128 horas (divididas en 64 horas presenciales y 64 horas a distancia.), distribuido en cuatro (4) módulos:

- Módulo 1. Conceptos Básicos de Estadística y de Investigación (16 horas).

- Módulo 2. Estadística Descriptiva (48 horas).

- Módulo 3. Contraste de hipótesis (48 horas).

- Módulo 4. Muestreos probabilísticos (16 horas).

La misión del diplomado es buscar la formación de estudiantes universitarios (pregrado y postgrado); además de la capacitación de profesionales en todas las áreas del saber, para la obtención de conocimientos en el manejo de programas informáticos de estadística que permitirá orientar la elaboración de trabajos de investigación basados 
en un enfoque cuantitativo. La visión es ser un Diplomado que aporte conocimientos de estadística pertinentes para realizar investigación en las diferentes áreas del saber de la Universidad de Los Andes y otras universidades nacionales e internacionales.

Los objetivos del diplomado planificado son:

- Analizar situaciones problema por medio de métodos estadísticos descriptivos con la ayuda del computador.

- Analizar situaciones problema por medio de métodos estadísticos inferenciales con la ayuda del computador.

- Calcular y determinar procedimientos de muestreo probabilísticos con aplicaciones informáticas.

El proceso educativo se llevó a cabo en clases teórico-prácticas de manera presencial bajo una teoría de aprendizaje constructivista, basada en la teoría de Dewey de aprender haciendo, la cual considera la necesidad de examinar el pensamiento a través de la acción si se quiere que este se transforme en conocimiento. Se prepararon actividades didácticas y materiales educativos que se elaboraron en el marco de un modelo instruccional de enseñanza directa, empleando como estrategias docentes la técnica de la pregunta y la resolución de problemas. Se utilizaron como herramientas de trabajo los programas Microsoft Excel, Epidat, SPSS para Windows, R y R-Studio. Las actividades a distancia se realizaron con tareas individuales que debían ser entregadas a través de Google Classroom. La evaluación de los contenidos se efectúa con seis (6) tareas divididas en 1) Conceptos básicos de estadística e investigación; 2) Tablas estadísticas; 3) Gráficos estadísticos; 4) Medidas descriptivas; 5) Contrastes de hipótesis; 6) Muestreo. A cada participante se le promedian las calificaciones y para obtener la certificación debe tener un mínimo de 15 puntos sin aproximaciones.

Es oportuno señalar que el proceso educativo b-learning, es decir, basados en el uso de las tecnologías Web como apoyo a la formación presencial, se adaptan perfectamente al modelo basado en la solución de problemas, buscando que el estudiante indague, analice, busque y organice la información con el fin de mostrar y desarrollar destrezas sobre el conocimiento que se quiere alcanzar (Sosa, García, Sánchez, Moreno y Reinoso, 2005).

De igual manera, es interesante aclarar que el uso de las tecnológicas de comunicación por medio de entornos virtuales que faciliten el aprendizaje, para que los estudiantes adquieran nuevas formas de interacción, requiere de una infraestructura tecnológica. Para el proceso de construcción de un diplomado, existen experiencias como el Diplomado Virtual Gestión para la Promoción de Salud, donde se realizó una investigación descriptiva, con la participación de médicos, enfermeras y pedagogos de 13 países iberoamericanos, se obtuvo $91 \%$ de aprobados y el $85 \%$ consideró beneficioso el modelo de aprendizaje en red (González, Vidal y Diego, 2013). 
Joan Chipia, Yorman Paredes.

En la construcción de experiencias en el área de la estadística a nivel de cursos o diplomados, se encontró el trabajo experimental aplicado a 33 estudiantes de un curso de Estadísticas Sociales en la Universidad Estatal de California, en el cual se dividieron aleatoriamente dos grupos, uno impartido en un aula tradicional y el otro grupo de manera virtual, los resultados cuantitativos mostraron que la clase virtual obtuvo un promedio de calificaciones de un $20 \%$ más alto que el puntaje de la clase tradicional en ambos exámenes. Además, los resultados posteriores a la prueba indican que la clase virtual tuvo un contacto entre pares y una percepción de más flexibilidad, comprensión del material y mayor afecto hacia las matemáticas, al final del semestre, que la clase tradicional (Schutte, 1997).

El trabajo de Baglin, Bedford y Bulmer (2013) resalta la importancia de un curso introductorio de estadística a nivel de postgrado de manera virtual, se propone aprender haciendo mediante una pregunta de investigación auto planteada mediante la recopilación y el análisis de datos utilizando los métodos cubiertos en el curso. La construcción de este proyecto se dividió en dos partes, una propuesta de mitad de semestre y una presentación en línea de fin de semestre. Tras la finalización de los proyectos, se les pidió a los estudiantes que proporcionaran comentarios cualitativos y cinco estudiantes participaron en entrevistas semiestructuradas. En conclusión, se determinó la existencia de facilidad de uso y contribución a la comprensión de la estadística. La retroalimentación cualitativa proporcionó información sobre cómo los proyectos pueden ayudar a desarrollar el pensamiento estadístico de los profesionales.

En los últimos años, en materia de educación se están adoptando nuevas tecnologías, entre ellas las aulas virtuales que les permiten a los docentes interactuar con los estudiantes en tiempo real. El aula virtual, es una forma sincrónica y asincrónica de elearning o b-learning, la cual ha sido adoptada por muchas organizaciones en su intento de promover el aprendizaje tratando de reducir los costos asociados con la capacitación presencial dirigida por un instructor (Sosa, García, Sánchez, Moreno y Reinoso, 2005). Las aulas virtuales han evolucionado y una reciente incorporación es Google Classroom, que es un sistema de gestión de aprendizaje para escuelas cuyo objetivo es simplificar la creación, distribución y evaluación de tareas.

Google Classroom es una plataforma que sirve para mejorar el flujo de actividades en un aula virtual; proporciona un conjunto de potentes funciones que lo convierten en una herramienta ideal para usar con los estudiantes; ayudando a ahorrar tiempo, mantener las clases organizadas y mejorar la comunicación con los alumnos; además de que permite aprovechar Google Docs, Drive y otras aplicaciones (Iftakhar, 2016). También esta plataforma tiene el potencial de racionalizar la comunicación y el flujo de trabajo para los estudiantes al proporcionar un solo punto de acceso a hilos de discusión y trabajo asignado. Puede ayudar a los discentes a mantener sus archivos más organizados porque todo su trabajo se puede almacenar sin papel en un solo programa. 
Algunas características de Google Classroom son: presenta una interfaz que facilita su uso, por su diseño simplista, con una comunicación continua con todo el curso a través de anuncios, correo electrónico y notificaciones push, permitiendo el ahorro de tiempo, ya que integra y automatiza el uso de otras aplicaciones de Google, incluidas documentos, diapositivas y hojas de cálculo, el proceso de administración de distribución de documentos, calificación, evaluación formativa y comentarios, está basado en la nube, es flexible y es gratis, simplemente registrándose con una cuenta de Google (Iftakhar, 2016). Es oportuno señalar que el profesor puede crear una clase y añadir a sus participantes directamente o bien les proporciona un código para que se apunten ellos mismos. Con un flujo de trabajo sencillo el profesor puede crear, asignar, revisar y señalar la nota a las tareas de forma ágil y desde una misma página sin necesidad de documentos en papel. El alumno puede ver todas las tareas en una página específica y todos los materiales de clase se archivan automáticamente en carpetas de Google Drive.

En vista de las consideraciones antes señaladas el objetivo de la investigación consiste en describir una experiencia educativa sobre la construcción de un Diplomado en Estadística Aplicada a la Investigación con la utilización de Google Classroom, Facultad de Medicina, Universidad de Los Andes, Mérida, Venezuela, 2018-2020.

\section{Método}

La investigación se realizó con un enfoque cuantitativo, tipo descriptivo, diseño no experimental de campo prospectiva (Hernández, Fernández y Baptista, 2014). Variable en estudio: calificación (puntos). Sujetos de estudio: estudiantes de siete cohortes del Diplomado en Estadística Aplicada a la Investigación, Facultad de Medicina, Universidad de Los Andes, Mérida, Venezuela, 2018-2020 ( $\mathrm{N=168).}$

Se utilizó como instrumento de recolección de datos el registro de calificaciones. El análisis estadístico empleado fue descriptivo por medio de indicadores absolutos (frecuencia) y relativos (porcentaje), además del cálculo de la media y desviación estándar con el programa Microsoft Excel 2019.

\section{Resultados}

Se han llevado a cabo siete (7) cohortes del Diplomado en Estadística Aplicada a la Investigación, con un total de 168 participantes, lo cual indica un promedio de 24 participantes por cohorte, la primera cohorte inició en julio de 2018 y la séptima culminó en marzo de 2020. En la Tabla 1 se describe que $83,3 \%(n=140)$ de los participantes inscritos en las siete cohortes, culminaron el Diplomado y el promedio ponderado de calificaciones fue de 16,98 puntos y la desviación estándar general fue de 1,473 puntos. 
Joan Chipia, Yorman Paredes.

Tabla 1. Calificaciones (puntos) de los participantes de las siete cohortes del Diplomado en Estadística Aplicada a la Investigación, Facultad de Medicina, Universidad de Los Andes, Mérida, Venezuela. 2018-2020.

\begin{tabular}{llllll}
\hline \multirow{2}{*}{ Cohortes } & \multicolumn{2}{l}{ No Participantes } & & & \\
\cline { 2 - 3 } & Iniciaron & Culminaron & Porcentaje & Promedio & $\begin{array}{c}\text { Desviación } \\
\text { Estándar }\end{array}$ \\
\hline I & 32 & 25 & 78,1 & 16,67 & 1,599 \\
II & 28 & 25 & 89,3 & 16,46 & 2,083 \\
III & 19 & 17 & 89,5 & 17,41 & 1,176 \\
IV & 21 & 15 & 71,4 & 17,27 & 1,280 \\
V & 8 & 8 & 100,0 & 18,63 & 0,518 \\
VI & 23 & 19 & 82,6 & 16,68 & 1,759 \\
VII & 37 & 31 & 83,8 & 17,05 & 1,209 \\
Total & 168 & 140 & 83,3 & 16,98 & 1,473 \\
\hline
\end{tabular}

Fuente: Cálculos propios.

\section{Discusión de resultados}

En la investigación científica basada en un enfoque cuantitativo, analizar datos y la construcción del razonamiento estadístico, son componentes esenciales, sin embargo, los profesionales o estudiantes de pregrado no lo tienen muy claro o desarrollado y por esta razón cursan el Diplomado en Estadística Aplicada a la Investigación, en el cual se utilizan programas estadísticos que son de utilidad, permitiendo aplicarla en sus trabajos de investigación o artículos.

A los estudiantes del diplomado se le concientizó sobre la naturaleza de aprender haciendo, es decir, de llevar a cabo el estudio de principio a fin, por ello, el docente requiere de compromiso con el aprendizaje de los participantes, porque debe supervisar, guiar y discutir estas actividades en clases, además de disponer de distintas herramientas informáticas y virtuales, que faciliten el proceso educativo como es el caso de Google Classroom. Se encontraron limitaciones de recursos y tiempo, por ello, la modalidad b-learning es de utilidad, debido a que se logró dar a conocer cómo utilizar los software informáticos de estadística planteados en el programa del Diplomado (Microsoft Excel, Epidat, SPSS para Windows, R y R-Studio) en la clase presencial, para posteriormente señalar asignaciones individuales, las cuales se plantearon con énfasis en las definiciones y procedimientos de estadística descriptiva e inferencial, para que los estudiantes tuvieran un contexto científico eficiente y se observen los conocimientos de manera aplicativa.

Es importante resaltar que las investigaciones de Schutte (1997), Baglin, Bedford y Bulmer (2013) y González, Vidal y Diego (2013) evidencian en sus experiencias virtuales resultados positivos, lo que es similar a los resultados obtenidos en el Diplomado en Estadística Aplicada a la Investigación, con la diferencia de que en la experiencia que está presentando, se hace en siete cohortes bajo la modalidad 
b-learning, para el aprendizaje de Estadística con programas informáticos y la tarea se entrega por medio de Google Classroom para reforzar lo aprendido en clases.

\section{Conclusiones}

La construcción del Diplomado en Estadística Aplicada a la Investigación con la utilización de Google Classroom es efectiva, pues el promedio de calificaciones y el porcentaje de culminación fue alto.

Se encontró que el proceso educativo bajo la modalidad b-learning mostró fluidez y permitió sortear dificultades logísticas, ahorrando tiempo, recursos y posibilitando que los participantes puedan madurar mejor las definiciones y procedimientos de estadística descriptiva e inferencial desarrollados en el Diplomado en Estadística Aplicada a la Investigación.

\section{Recomendaciones}

Diseñar el Diplomado en Estadística Aplicada a la Investigación bajo la modalidad elearning, para posteriormente elaborar otra experiencia educativa, en la cual se comparen las dos modalidades (e-learning, b-learning).

\section{Referencias}

Baglin, J., Bedford, A., \& Bulmer, M. (2013). Students' Experiences and Perceptions of Using a Virtual Environment for Project-Based Assessment in an Online Introductory Statistics Course. Technology Innovations in Statistics Education, 7(2), 1-16. Recuperado de: https://escholarship.org/uc/item/137120mt Consulta: 14-03-2020.

Chipia, J. (2013). Efectividad de un programa de enseñanza/aprendizaje sobre estadística descriptiva utilizando Calc de Open Office. VII Congreso Iberoamericano de Educación Matemática, Montevideo, Uruguay, 16-20 de noviembre de 2013.

Chipia, J. (2014). Experiencia pedagógica de construcción de un blog por estudiante. En Contreras, J., Batanero, C., Godino, J., Cañadas, G., Arteaga, P., Molina, E., Gea, M. y López, M. (Eds.). Didáctica de la Estadística, Probabilidad y Combinatoria, 2 (pp. 317-323). Granada, 10-12 de abril de 2015.

Chipia, J. (2017). Ontología de la Bioestadística. GICOS, 2(2), 3

Chipia, J. y Paredes, Y. (2017). Proyectos: medio integrador en el aprendizaje de bioestadística. Paradigma, 38(2), 334-345

Chipia, J., Cadenas, R. y Lara, C. (2012). Propuesta para la enseñanza de organización de datos para variables cualitativas. EDUCERE, 16(53), 185-196.

González, B.; Vidal, M. y Diego, F. (2013). Experiencia cubana sobre el Diplomado en Promoción de salud en el Campus virtual de la Salud Pública. Educación Médica Superior, 27(1), 12-24. Recuperado de 
http://scielo.sld.cu/scielo.php?script=sci_arttext\&pid=S0864-

21412013000100003\&Ing=es\&tIng=es Consulta: 14-03-2020

Iftakhar, S. (2016). Google Classroom: What works and how? Journal of Education and Social Sciences, 3, 12-18. Recuperado de http://jesoc.com/wpcontent/uploads/2016/03/KC3_35.pdf Consulta: 15-03-2020

Schutte, J. (1997). Virtual teaching in higher education: The new intellectual superhighway or just another traffic jam? California: California State University, Northridge.

Sosa, R., García, A., Sánchez, J., Moreno, P. y Reinoso, J. (2005). B-learning y teoría del aprendizaje constructivista en las disciplinas informáticas: un esquema de ejemplo a aplicar. Recent Research Developments in Learning Technologies (en línea). Recuperado de: http://brd.unid.edu.mx/b-learning-y-teoria-del-aprendizajeconstructivista-en-las-disciplinas-informaticas-un-esquema-de-ejemplo-a-aplicar/ Consulta: $25-03-2020$

Universidad de Los Andes (2011). Reglamento de Programas de Formación, Actualización y Capacitación de la Universidad de Los Andes. Mérida: Autor. Recuperado de http://web.ula.ve/viceacademico/reglamentos-e-informes/

Consulta: $10-03-2020$ 